\title{
THE SHAPE OF THE EMERGING CONDENSATE IN EFFECTIVE MODELS OF CONDENSATION
}

\author{
VOLKER BETZ, STEFFEN DEREICH AND PETER MÖRTERS
}

\begin{abstract}
We consider effective models of condensation where the condensation occurs as time $t$ goes to infinity. We provide natural conditions under which the build-up of the condensate occurs on a spatial scale of $1 / t$ and has the universal form of a Gamma density. The exponential parameter of this density is determined only by the equation and the total mass of the condensate, while the power law parameter may in addition depend on the decay properties of the initial condition near the condensation point. We apply our results to some examples, including simple models of Bose-Einstein condensation.
\end{abstract}

Keywords: Emergence, kinetic equation, qunatum particles, Bose-Einstein, House-ofcards model, selection, mutation, singular solution, non-linear partial differential equation, non-equilibrium phenomena.

2010 Math. Subj. Class.: 35F25 (primary); 82C05; 82C10; 82C26; 35Q40; 82C40.

\section{Motivation And BACKGROUnd}

Condensation is an important and interesting phenomenon, which is present in many different physical systems. Loosely speaking, condensation occurs when for a system of many particles, a given relevant quantity has the same value for a macroscopic fraction of those particles. The examples that are relevant to this article are natural selection and mutation, where the particles are individuals, the relevant quantity is the fitness, and the condensation occurs at the maximal fitness; and Bose-Einstein condensation, where the particles are Bosons, the relevant quantity is the energy, and the condensation occurs at the minimal quantum energy level. We are interested in what a condensing system looks like when it is dynamically close to condensation.

We will not deal with the difficult issue of condensation in many-particle systems itself, but will instead investigate effective models and manifestations of condensation in a scalar variable. These models are then given in the form of non-linear measure-valued equations in $1+1$ dimensions, the first of the variables being time, and the other the scalar quantity mentioned above. More precisely, for $t \in \mathbb{R}_{0}^{+}$, the finite measure $p_{t}$ describes the concentration of particles at relevant quantity $x$, and $\left(p_{t}\right)_{t \geqslant 0}$ solves the equation

$$
\partial_{t} p_{t}(\mathrm{~d} x)=F\left(x, p_{t}\right) .
$$

with some functional $F$. We will give three concrete examples of such systems below: Kingman's model of selection and mutation [11, an approximate model for Bose- Einstein condensation due to Buffet, de Schmedt and Pulé [1] (henceforth called the BSP-model), and a model for bosons in a heat bath investigated by Escobedo, Mischler and Velazquez [7, 8, 9] (referred to als EMV-model below). Another effective model of condensation is the Boltzmann-Nordheim equation [12, 13, 15, 10, but as we will see below, it is too singular for our theory to apply. 
In the effective models, condensation is characterized by the behavior of $p_{t}$ as $t \rightarrow t^{*}$, where $t^{*} \leqslant \infty$ is the time at which condensation occurs. Typically, one assumes that the initial condition $p_{0}$ is absolutely continuous with respect to the Lebesgue measure, and $t^{*}$ is the infimum over all times where $p_{t}(\mathrm{~d} x)$ is not absolutely continuous with respect to the Lebesgue measure. If one finds that there exist $\rho>0$ and $q \in L^{1}$ so that for suitable test functions $\phi$,

$$
\lim _{t \uparrow t^{*}} \int p_{t}(\mathrm{~d} x) \phi(x)=\rho \phi\left(x^{*}\right)+\int q(x) \phi(x) \mathrm{d} x,
$$

this is paraphrased by saying that condensation occurs at $x=x^{*}$, at time $t=t^{*}$. We then refer to $\rho$ as the mass of the condensate, while $q$ is the bulk density. The approach to the limit in Equation (1.2) can be interpreted as the formation of an approximate Dirac distribution around $x^{*}$ when $t$ is near $t^{*}$. The topic of our paper is to investigate in the case $t^{*}=\infty$ the asymptotic shape of this approximate Dirac distribution in the correct scale.

In cases where $t^{*}<\infty$, much less is known. The best results that we are aware of are those by Escobedo and Velazquez for the Boltzmann-Nordheim equation [10]. They show that under suitable assumptions on the mass of the initial condition, the solution $p_{t}(\mathrm{~d} x)$ to (1.1) has a Lebesgue density $\tilde{p}_{t}$ that explodes in finite time, i.e. there exists a $t_{*}^{-}<\infty$ so that

$$
\liminf _{t \uparrow t_{*}^{-}}\left\|\tilde{p}_{t}\right\|_{\infty}=\infty
$$

They also show that there exists a $t_{*}^{+} \geqslant t_{*}^{-}$so that the weak solution of (1.1) contains a Dirac mass at the condensation point $x^{*}=0$ for some $t \in\left[t_{*}^{-}, t_{*}^{+}\right]$. What they cannot show is that the infimum over all possible $t_{*}^{+}$equals $t_{*}^{-}$, and so an equation like (1.2) is currently not known. We are not aware of any natural examples where (1.2) has been proved for some $t^{*}<\infty$, and therefore stick to the case $t^{*}=\infty$.

To our knowledge, the following rigorous results about the asymptotic shape of such emerging condensates exist: In [5], it is shown that for Kingman's model of selection and mutation, the shape is the one of a Gamma-distribution. The same result is found in [9] for a special case of the EMV-model. They also find the influence of the initial condition, depending on its behavior near the condensation point, that will appear in our results below. The results rely on explicit solution formulas for all times, although in [9] a formal asymptotic expansion is used in order to cover also cases where such a solution formula is missing.

The contribution of our paper is to provide conditions which are easy to check, do not require the knowledge of a solution formula, and are sufficient to conclude both the Gamma shape of the near condensate and the possible dependence of that shape on the initial condition. Our conditions are natural in that they only require knowledge about the right hand side of (1.2) when $p_{t}$ is very close to the formal stationary solution $\rho \delta_{x^{*}}+q$. Precise statements follow in the next section.

Acknowledgements: The authors would like to thank Daniel Ueltschi for many fruitful discussions and J.J.L. Velazquez for useful comments on the Boltzmann-Nordheim equation, and for pointing out reference [9].

\section{Assumptions And main Result}

We treat models where condensation occurs at the boundary of the set of possible values of the scalar quantity $x$, and we normalize these models so that this value is $x=0$. Further we restrict attention to the case where $t^{*}=\infty$. Let $\mathcal{M}_{0}:=\left\{\rho \delta_{0}+p \mathrm{~d} x: \rho \geqslant 0, p \in L^{1}\left(\mathbb{R}_{0}^{+}\right)\right\}$ 
be the subspace of the space of finite measures on $\mathbb{R}_{0}^{+}$that have a density with respect to Lebesgue measure except possibly at the origin where we allow for a Dirac measure $\delta_{0}$ with mass $\rho$. Fix $\alpha>0$, and let $\boldsymbol{B}: \mathcal{M}_{0} \rightarrow C\left(\mathbb{R}_{0}^{+}\right)$and $\boldsymbol{C}: \mathcal{M}_{0} \rightarrow C\left(\mathbb{R}_{0}^{+}\right)$be (not necessarily linear) operators. Consider the equation

$$
\partial_{t} p_{t}(\mathrm{~d} x)=\boldsymbol{B}\left[p_{t}\right] p_{t}(\mathrm{~d} x)+x^{\alpha} \boldsymbol{C}\left[p_{t}\right] \mathrm{d} x .
$$

An element $p \in \mathcal{M}_{0}$ is called stationary, if

$$
\boldsymbol{B}[p] p(\mathrm{~d} x)+x^{\alpha} \boldsymbol{C}[p] \mathrm{d} x=0 .
$$

In most cases we consider evolutions where $p_{t}$ does not have an atom in 0 . In this case we refer to the Lebesgue density of $p_{t}$ by the same symbol $p_{t}$. With this convention the equation reads

$$
\partial_{t} p_{t}(x)=\boldsymbol{B}\left[p_{t}\right] p_{t}(x)+x^{\alpha} \boldsymbol{C}\left[p_{t}\right] .
$$

When comparing (2.1) to the most general equation (1.2), we see that we demand a decomposition of the right hand side into a homogenous part and a remainder. While such a decomposition can always be achieved (e.g. by setting $\boldsymbol{B}=0$ ), the restriction lies in the assumed regularity of the images under $\boldsymbol{B}$ and $\boldsymbol{C}$. In particular, we assume that when dividing the inhomogenous part by a factor of $x^{\alpha}$, we still retain a function that is bounded at $x=0$. In all the concrete and relevant examples that we are aware of, the decomposition is unique and easy to find. In the Boltzmann-Nordheim model, however, measures with a Dirac mass at the origin are too singular for the equation to make sense in a classical way [10], and so a decomposition like (2.1) with the corresponding regularity assumptions fails.

Definition: We say that a solution $\left(p_{t}\right)_{t \geqslant 0}$ to (2.1) converges regularly to an element $p_{\infty} \in \mathcal{M}_{0}$ if

(i): $p_{t} \rightarrow p_{\infty}$ weakly as $t \rightarrow \infty$ as measures;

(ii): the following two equations hold:

$$
\begin{gathered}
\lim _{t \rightarrow \infty}\left\|\boldsymbol{B}\left[p_{t}\right]-\boldsymbol{B}\left[p_{\infty}\right]\right\|_{C^{1}([0, \delta])}=0, \\
\lim _{t \rightarrow \infty}\left\|\boldsymbol{C}\left[p_{t}\right]-\boldsymbol{C}\left[p_{\infty}\right]\right\|_{C([0, \delta])}=0 .
\end{gathered}
$$

Here, $\|f\|_{C^{1}([0, \delta])}=\sup \left\{|f(x)|+\left|f^{\prime}(x)\right|: 0 \leqslant x \leqslant \delta\right\}$, and $\|f\|_{C([0, \delta])}=\sup \{|f(x)|$ : $0 \leqslant x \leqslant \delta\}$.

When $p_{\infty}$ is a measure with positive condensate mass, (i) above is what is usually proved when condensation is shown, see e.g. [8, 1]. (ii) is more particular to our needs. Note that (2.2) demands that the difference of $\boldsymbol{B}\left[p_{t}\right]$ and $\boldsymbol{B}\left[p_{\infty}\right]$ is of higher regularity than each individual term needs to be.

In two of the examples that we will give, the operators $\boldsymbol{B}$ and $\boldsymbol{C}$ are affine integral operators. In both of those examples, it has been shown that the convergence of $p_{t}$ to $p_{\infty}$ is in $L^{1}$ away from $x=0$. The next proposition states that in such cases, (2.2) and (2.3) already follow from natural regularity assumptions on the integral kernels of $\boldsymbol{B}$ and $\boldsymbol{C}$.

Proposition 2.1. Assume that $\boldsymbol{B}$ and $\boldsymbol{C}$ are affine integral operators, i.e.

$$
\boldsymbol{B}[p](x)=\int_{0}^{\infty} K_{b}(x, y) p(\mathrm{~d} y)+R_{b}(x), \quad \boldsymbol{C}[p](x)=\int_{0}^{\infty} K_{c}(x, y) p(\mathrm{~d} y)+R_{c}(x),
$$

where $K_{b}, K_{c}$, and $\partial_{x} K_{b}$ are elements of $C_{b}\left([0, \delta] \times \mathbb{R}_{0}^{+}\right)$for some $\delta>0$, while $R_{b}$ and $R_{c}$ are arbitrary functions. 
Let $\left(p_{t}\right)$ be a solution to (2.1) with initial condition $p_{0}(x) \mathrm{d} x$, i.e. without an atom at zero. Assume that $p_{t}$ converges weakly to $p_{\infty}=\rho \delta_{0}+q(x) \mathrm{d} x$ with $\rho \geqslant 0$ and $q \in L^{1}$, and in addition assume that

$$
\lim _{t \rightarrow \infty} \int_{\delta}^{\infty}\left|p_{t}(x)-q(x)\right| \mathrm{d} x=0
$$

for all $\delta>0$. Finally, assume that there exists $\tilde{\delta}>0$ such that

$$
\limsup _{t \rightarrow \infty} \int_{0}^{\tilde{\delta}}\left|p_{t}(x)\right| \mathrm{d} x<\infty .
$$

Then $p_{t}$ converges regularly to $p_{\infty}$.

The proof consists of standard applications of integral convergence theorems. We give it in the appendix for the convenience of the reader. Note that since $R_{b}$ and $R_{c}$ drop out when considering differences like $\boldsymbol{B}\left[p_{t}\right](x)-\boldsymbol{B}\left[p_{\infty}\right](x)$, they are indeed arbitrary, although the regularity requirements preceding (2.1) will usually mean that they need to be continuous and bounded.

Now we state our main result.

Theorem 2.2. Assume that $\left(p_{t}\right)$ solves equation (2.1) with initial condition $p_{0}(x) \mathrm{d} x$, i.e. without atom at zero. For the density $p_{0}$, assume that there exists $\alpha_{0}>0$ and a function $\eta: \mathbb{R}_{0}^{+} \rightarrow \mathbb{R}_{0}^{+}$, which is continuous and positive at zero, so that

$$
p_{0}(x):=x^{\alpha_{0}} \eta(x) .
$$

Assume further that $\left(p_{t}\right)$ converges regularly to a stationary limit $p_{\infty}=\rho \delta_{0}+q(x) \mathrm{d} x$ with $\rho>0$ and $q \in L^{1}$. Finally, assume that for this limit,

$$
c_{1}:=\boldsymbol{C}\left[p_{\infty}\right](0)>0,
$$

and that, for some $\alpha>0$,

$$
c_{2}:=\lim _{x \rightarrow 0} \frac{x^{\alpha-1}}{q(x)}>0 \text { exists. }
$$

Then with $\gamma:=c_{1} c_{2}$ and $\beta:=\min \left\{\alpha, \alpha_{0}\right\}$ we have, uniformly on compact intervals of $\mathbb{R}_{0}^{+}$, that

$$
\lim _{t \rightarrow \infty} \frac{1}{t} p_{t}\left(\frac{x}{t}\right)=C \mathrm{e}^{-\gamma x} x^{\beta} .
$$

Above, $C=\rho \gamma^{\beta} / \Gamma(\beta)$, i.e. $C$ is such that the right hand side of (2.8) integrates to $\rho$.

The main feature of this result is the universal nature of the gamma shape of the emerging condensate. In all examples that we are aware of, the stationary limit $p_{\infty}$ in Theorem 2.2 only depends on the mass of the initial condition, but not on its shape. In these cases, the statement can also be read as the following dichotomy: either $\alpha \leqslant \alpha_{0}$, in which case the initial condition is irrelevant for the shape of the emerging condensate; or, $\alpha_{0}<\alpha$, in which case the exponential decay of the Gamma distribution is still governed by the constant from (2.7), but the power law near $x=0$ is the same as in the initial condition. From the calculations in our proofs, it is apparent that the second case could be strengthened in the following way: if the initial condition dominates the inhomogeniety near $x=0$, the emerging condensate will look like the initial condition at an appropriate scale. In particular, one might think of initial conditions that switch between different power laws $\alpha_{1}$ and $\alpha_{2}$ infinitely often in the approach to zero. In that case, we would have no convergence in (2.8), but rather an oscillating behavior. Since this case does not seem very relevant and would need rather careful statements and investigations, we do not pursue it any further. 
Note that condition (2.7) can alternatively be read as a condition on $\boldsymbol{B}\left[p_{\infty}\right](x)$ near the point $x=0$. Namely, by stationarity of $p_{\infty}$ we have that

$$
\boldsymbol{B}\left[p_{\infty}\right](x)=-\frac{x^{\alpha}}{q(x)} \boldsymbol{C}\left[p_{\infty}\right](x)
$$

for Lebesgue almost all $x \geqslant 0$ and by continuity this holds for all $x \geqslant 0$. For $x=0$ the right hand side equals zero so that $\boldsymbol{B}\left[p_{\infty}\right](0)=0$. Thus, condition (2.7) means that $x \mapsto \boldsymbol{B}\left[p_{\infty}\right](x)$ is differentiable at $x=0$, and that its derivative equals $-\gamma$.

\section{EXAMPLES}

3.1. Selection mutation equations. A natural set of examples for our theory arise from equations describing the fitness distribution of a population evolving by selection and mutation. We focus on Kingman's model [11] and briefly mention some generalizations and variants at the end of this section.

Kingman's model of selection and mutation is originally framed in discrete time, see [11]. We start with an initial fitness distribution $p_{0}(\mathrm{~d} x)$ of a diffuse population, which is a probability measure on $(0,1)$. By $p_{n}(\mathrm{~d} x)$ we denote the fitness distribution in the $n$-th generation. It satisfies the recursion

$$
p_{n+1}(\mathrm{~d} x)=(1-\beta) \frac{x}{w_{n}} p_{n}(\mathrm{~d} x)+\beta r(\mathrm{~d} x),
$$

where $w_{n}=\int_{0}^{1} x p_{n}(\mathrm{~d} x)$ is the mean fitness at generation $n, r$ is the fitness distribution for spontaneous mutations, and $\beta \in(0,1)$ is the frequency of mutation. We assume that $r$ is a probability measure on $(0,1)$ with essential supremum at $x=1$. Kingman's idea is that a proportion $1-\beta$ of the population is selected from the previous generation with a selective advantage proportional to their fitness, and a proportion $\beta$ of the population experiences mutation, which destroys the individuals' biochemical 'house of cards' so that the mutant fitness distrbution $r$ does not depend on their previous fitness. Kingman showed that condensation at the maximal fitness $x=1$ occurs if

$$
\beta \int_{0}^{1} \frac{r(\mathrm{~d} x)}{1-x}<1 .
$$

To adapt the model to our framework, we switch to a continuous time model and change variables so that condensation occurs at $x=0$. The result is the equation

$$
\partial_{t} p_{t}(\mathrm{~d} x)=\left((1-\beta) \frac{1-x}{w\left[p_{t}\right]}-1\right) p_{t}(\mathrm{~d} x)+\beta u(\mathrm{~d} x),
$$

where $w\left[p_{t}\right]=\int_{0}^{1}(1-x) p_{t}(\mathrm{~d} x)$ and $u(\mathrm{~d} x)=r(\mathrm{~d}(1-x))$. Kingman's arguments show that if

$$
\beta \int_{0}^{1} \frac{u(\mathrm{~d} x)}{x}<1
$$

we have $p_{t} \rightarrow p_{\infty}$ weakly for the stationary solution $p_{t}$ given by

$$
p_{\infty}(\mathrm{d} x)=\left(1-\beta \int_{0}^{1} \frac{u(\mathrm{~d} x)}{x}\right) \delta_{0}+\beta \frac{u(\mathrm{~d} x)}{x} .
$$

We assume that $u(x)=x^{\alpha} u_{0}(x)$, for some $\alpha>0$, and $u_{0}(x)$ continuous and strictly positive at $x=0$. Then,

$$
\boldsymbol{B}[p]=(1-\beta) \frac{1-x}{w[p]}-1,
$$


and $\boldsymbol{C}[p]=\beta u_{0}$. In particular (2.2) and (2.3) are trivially fulfilled. Moreover,

$$
w\left[p_{\infty}\right]=\left(1-\beta \int_{0}^{1} \frac{u(\mathrm{~d} x)}{x}\right)+\beta \int \frac{1-x}{x} u(\mathrm{~d} x)=1-\beta,
$$

as $u(\mathrm{~d} x)$ is a probability measure. Then, $\boldsymbol{B}\left[p_{\infty}\right](x)=-x$, and $\boldsymbol{C}\left[p_{\infty}\right](x)=\beta u_{0}(x)$, confirming that $p_{\infty}$ is stationary. Moreover, the bulk density $q(x)$ is given by $\beta u(x) / x=$ $\beta x^{\alpha-1} u_{0}(x)$. Thus, $c_{2}$ from (2.7) is given by $1 / \beta u_{0}(0)$, and Theorem 2.2 holds with $\gamma=1$. We thus obtain the gamma-shape of the emerging condensate under less restrictive conditions than Dereich and Mörters [5].

Kingman's model was generalized by Yuan [16] to model the long-term evolution of Eschrichia.coli in the Lenski experiment. This model can still be fitted to our framework. Park and Krug [14] generalize Kingman's model to unbounded fitness distributions, which leads to a qualitatively different emergence of a condensate (at infinity). They also investigate the relation of Kingman's model to a stochastic finite population model. Dereich 4 identifies the shape of the emergent condensate in a random network model with fitness.

We now discuss in more detail a stochastic particle model based on a branching process closely related to Kingman's original model, which is investigated in [6]. In this model immortal particles produce offspring with a rate given by their fitness. Independently, each offspring particle is a mutant with probability $\beta$ and otherwise inherits the parent's fitness. Mutants receive their fitness by sampling from the distribution $r$. The expected fitness distribution $q_{t}$ of particles alive at time $t$ therefore satisfies

$$
\dot{q}_{t}(\mathrm{~d} x)=(1-\beta) x q_{t}(\mathrm{~d} x)+\beta \int y q_{t}(\mathrm{~d} y) r(\mathrm{~d} x) .
$$

Taking $a_{t}=\int_{0}^{t} \int y q_{s}(\mathrm{~d} y) \mathrm{d} s$ the normalized and time-changed quantity

$$
\tilde{p}_{t}=\frac{q_{a_{t}^{-1}}}{\int q_{a_{t}^{-1}}(\mathrm{~d} y)}
$$

therefore satisfies

$$
\dot{\tilde{p}}_{t}(\mathrm{~d} x)=\left((1-\beta) \frac{x}{\int y \tilde{p}_{t}(\mathrm{~d} y)}-1\right) \tilde{p}_{t}(\mathrm{~d} x)+\beta r(\mathrm{~d} x) .
$$

Moving now the condensation point to the origin we are back in Kingman's model and Theorem 2.1 can be applied. If (3.1) holds we have $a_{t} \sim(1-\beta) t$ and we observe that also in the stochastic model the condensate is forming on the scale $1 / t$ in expectation. The behaviour of this stochastic model in probability is more difficult to investigate and largely open.

3.2. A model of Bosons in contact with a bath of Fermions. In a series of papers [7, 8, 9, Escobedo, Mischler and Velazquez study an effective model for Bosons in contact with a bath of Fermions in thermal equilibrium. Given a function $b: \mathbb{R}_{+}^{0} \times \mathbb{R}_{+}^{0} \rightarrow \mathbb{R}_{+}$of the form

$$
b(x, y)=\mathrm{e}^{\eta x} \mathrm{e}^{\eta y} \sigma(x, y)
$$

with $\eta \in[0,1)$ and $0 \leqslant \sigma \in L^{\infty}\left(\mathbb{R}_{+}^{0} \times \mathbb{R}_{+}^{0}\right)$ symmetric, one asks for solutions $\left(F_{t}\right)_{t \geqslant 0}$ of the equation

$$
\partial_{t} F_{t}(x)=\int_{0}^{\infty} b(x, y)\left(F_{t}(y)\left(x^{2}+F_{t}(x)\right) e^{-x}-F_{t}(x)\left(y^{2}+F_{t}(y)\right) e^{-y}\right) \mathrm{d} y .
$$


Note that the right hand side is well defined as long as $F_{t}$ is in the space $L^{1}\left(\mathbb{R}_{0}^{+}, \mathrm{e}^{\eta x} \mathrm{~d} x\right)$. To apply our results we consider the transformed solution $\left(p_{t}\right)_{t \geqslant 0}$ given by $p_{t}(x)=\mathrm{e}^{\eta x} F_{t}(x)$. It satisfies as $L^{1}\left(\mathbb{R}_{+}^{0}\right)$-valued system the equation

$$
\partial_{t} p_{t}(x)=\mathrm{e}^{\eta x} \int_{0}^{\infty} \sigma(x, y)\left(p_{t}(y)\left(x^{2} \mathrm{e}^{\eta x}+p_{t}(x)\right) \mathrm{e}^{-x}-p_{t}(x)\left(y^{2} \mathrm{e}^{\eta y}+p_{t}(y)\right) \mathrm{e}^{-y}\right) \mathrm{d} y .
$$

The extension of this equation to elements of $\mathcal{M}_{0}$ is straightforward, and it fits into the framework of (2.1) with $\alpha=2$,

$$
\boldsymbol{B}[p](x)=\mathrm{e}^{\eta x} \int_{0}^{\infty} p(\mathrm{~d} y) \sigma(x, y)\left(\mathrm{e}^{-x}-\mathrm{e}^{-y}\right)-\mathrm{e}^{\eta x} \int_{0}^{\infty} \sigma(x, y) y^{2} \mathrm{e}^{(\eta-1) y} \mathrm{~d} y,
$$

and

$$
\boldsymbol{C}[p](x)=\mathrm{e}^{(2 \eta-1) x} \int_{0}^{\infty} \sigma(x, y) p(\mathrm{~d} y)
$$

Under suitable assumptions on $\sigma$ and the initial condition, Escobedo and Mischler 7 , 8] show existence of solutions for all times, and convergence of the solution as $t \rightarrow \infty$ towards the stationary solution. In the case where $\int_{0}^{\infty} F_{0}(x) \mathrm{d} x>\int_{0}^{\infty} \frac{x^{2}}{\mathrm{e}^{x}-1} \mathrm{~d} x=2.40411 \ldots$ condensation occurs and the limit is of the form

$$
F_{\infty}(\mathrm{d} x)=\rho \delta_{0}+Q(x) \mathrm{d} x \quad \text { with } Q(x)=\frac{x^{2}}{\mathrm{e}^{x}-1}
$$

and $\rho=\int_{0}^{\infty} F_{0}(x) \mathrm{d} x-\int \frac{x^{2}}{\mathrm{e}^{x}-1} \mathrm{~d} x$. In that case $\left(p_{t}\right)$ has as limit

$$
p_{\infty}(\mathrm{d} x)=\rho \delta_{0}+q(x) \mathrm{d} x \quad \text { with } q(x)=\frac{x^{2} \mathrm{e}^{\eta x}}{\mathrm{e}^{x}-1} .
$$

In 9], the authors investigate the shape of the emerging condensate; they show rigorously (by means of an explicit solution formula) that the emerging condensate is Gamma-shaped in the special case that $b(x, y) \equiv 1$. Moreover, they find that the power law parameter of the Gamma distribution depends on how the initial condition vanishes at $x=0$; this corresponds to Theorem 2.2 of the present paper. Very interestingly, they also obtain asymptotics for the case where the initial condition already has a Dirac mass at $x=0$. In that case, there is no emerging condensate as $t \rightarrow \infty$, instead the Dirac mass itself grows to its final value (determined by the total mass of the initial condition) as $t \rightarrow \infty$; see Theorem 1, part (ii) of [9]. This result shows that we cannot drop the assumption on absolute continuity of the initial condition in Theorem 2.2 .

We will now use our general theory in order to find more general conditions on $b$ so that the Gamma shape of the emerging condensate still holds. We first assume that $(x, y) \mapsto \sigma(x, y)$ is continuous, continuously differentiable with respect to $x$ for all $y$, and that, for some $\delta>0$,

$$
\sup \left\{\left|\partial_{x} \sigma(x, y)\right|: 0 \leqslant x \leqslant \delta, y \in \mathbb{R}^{+}\right\}<\infty .
$$

This condition is tailor-made for the assumptions on $\boldsymbol{B}$ and $\boldsymbol{C}$ of Proposition 2.1. In fact it is slightly more than we really need, since we have

$$
K_{b}(x, y)=\sigma(x, y)\left(\mathrm{e}^{-x}-\mathrm{e}^{-y}\right),
$$

and thus at $x=y$ differentiability of $\sigma$ is not needed due to the presence of the factor $\mathrm{e}^{-x}-\mathrm{e}^{-y}$. We further assume that

$$
\int_{0}^{\infty} \sigma(0, y) p_{\infty}(\mathrm{d} y)>0
$$


This assumption ensures the validity of conditions (2.6) and (2.7). Indeed, we compute

$$
\boldsymbol{C}\left[p_{\infty}\right](x)=\mathrm{e}^{(2 \eta-1) x}\left(\rho \sigma(x, 0)+\int_{0}^{\infty} \sigma(x, y) \frac{y^{2} \mathrm{e}^{\eta y}}{\mathrm{e}^{y}-1} \mathrm{~d} y\right),
$$

and thus $\boldsymbol{C}\left[p_{\infty}\right](0)>0$, so $(2.6)$ holds. Since

$$
\lim _{x \rightarrow 0} \frac{x^{\alpha-1}}{q(x)}=\lim _{x \rightarrow 0} \mathrm{e}^{\eta x} \frac{\mathrm{e}^{x}-1}{x}=1,
$$

we also have (2.7), and find that $\gamma=\int \sigma(0, y) p_{\infty}(\mathrm{d} y)$. Note that (3.8) is weaker than the assumption $\sigma(0,0)>0$ that was made in the appendix of [9], where non-constant $b$ are treated non-rigorously, using matched asymptotic expansions.

Finally we need to verify (2.4). This is proved by Escobedo and Mischler in Theorem 6 of [8] for the case $\eta=0$ and we believe that the arguments given there allow to prove (2.4) also for arbitrary values of $\eta>0$. In conclusion, if conditions (3.7) and (3.8) hold and $\eta=0$, then Theorem 2.2 holds with $\alpha=2$ and $\gamma=\int \sigma(0, y) p_{\infty}(\mathrm{d} y)$.

3.3. Kinetics of Bose-Einstein condensation. Our final example is a simple model for the emergence of a condensate in a Bose gas in contact with a heat bath, which was developed by Buffet, de Smedt and Pulé in [1, 2].

Let $\hat{C}: \mathbb{R} \rightarrow \mathbb{R}_{0}^{+}$be a strictly positive, bounded function satisfying the KMS relation

$$
\hat{C}(-z)=\hat{C}(z) \mathrm{e}^{\beta z}
$$

for some $\beta>0$. Physically, $\hat{C}$ is the Fourier transform of the heat bath correlation function, and $\beta>0$ is the inverse temperature of the heat bath. Note also that the assumption that $\hat{C}$ is bounded and (3.9) imply exponential decay of $\hat{C}(z)$ as $z \rightarrow \infty$.

We also define

$$
\begin{aligned}
& A(z)=\hat{C}(-z)-\hat{C}(z)=\hat{C}(z)\left(\mathrm{e}^{\beta z}-1\right), \\
& F(x)=\frac{x^{1 / 2}}{\sqrt{2} \pi^{2}} .
\end{aligned}
$$

$F$ is the density of states in the case of a Bose gas in a 3-dimensional box. In order to model the Bose gas in other environments we would need to modify $F$; for example, for the Bose gas in a 3 -dimensional harmonic trap, $F$ would decay like $x^{3 / 2}$ near $x=0$; see also the discussion in [3]. In what follows we will consider the more general form

$$
F(x)=f(x) x^{\alpha}, \quad \alpha>0, \quad f(0)>0 .
$$

The energy distribution $p_{t}$ of the Bose gas at time $t \geqslant 0$ then satisfies the equation

$$
\begin{aligned}
\partial_{t} p_{t}(x)=\int_{0}^{\infty} & A(y-x) p_{t}(x) p_{t}(y) \mathrm{d} y \\
& -\int_{0}^{\infty} \hat{C}(y-x) F(y) p_{t}(x) \mathrm{d} y+\int_{0}^{\infty} \hat{C}(x-y) p_{t}(y) F(x) \mathrm{d} y .
\end{aligned}
$$

It is easy to check that (3.11) preserves the total mass $\int_{0}^{\infty} p_{t}(x) \mathrm{d} x$ for all times $t \geqslant 0$. It has been shown in [1] that there is a global solution to (3.11) and moreover, there exists $\rho_{\mathrm{c}}>0$ such that, for all initial energy densities satisfying

$$
\int_{0}^{\infty} p_{0}(x) \mathrm{d} x>\rho_{\mathrm{c}}+\rho
$$


for some $\rho>0,\left(p_{t}\right)$ converges weakly to the stationary solution

$$
p_{\infty}(\mathrm{d} x)=\rho \delta_{0}+q(x) \mathrm{d} x
$$

with bulk density

$$
q(x)=\frac{F(x)}{\mathrm{e}^{\beta x}-1} .
$$

Moreover, Theorem 2 of [1] states that

$$
\lim _{t \rightarrow \infty} \int_{c}^{\infty}\left|p_{t}(y)-q(y)\right| \mathrm{d} y=0
$$

for all $c>0$. Thus, (2.4) and (2.5) hold.

The decomposition of equation (3.11) according to (2.1) is given by

$$
\begin{aligned}
& \boldsymbol{B}[p](x)=\int_{0}^{\infty} A(y-x) p(y) \mathrm{d} y-\int_{0}^{\infty} \hat{C}(y-x) F(y) \mathrm{d} y, \\
& \boldsymbol{C}[p](x)=f(x) \int_{0}^{\infty} \hat{C}(x-y) p(y) \mathrm{d} y .
\end{aligned}
$$

So in the context of Proposition 2.1. we have $K_{b}(x, y)=A(y-x), K_{c}(x, y)=\hat{C}(x-y)$, $R_{1}(x)=\int_{0}^{\infty} \hat{C}(y-x) F(y) \mathrm{d} y$ and $R_{2}(x)=0$.

We now assume that the restriction of $\hat{C}$ to the interval $(-\infty, 0)$ is continuously differentiable with a bounded derivative, and that $f$ is continuous. Then the integral kernels $K_{b}(x, y)=A(y-x)$ and $K_{c}(x, y)=\hat{C}(x-y)$ are bounded and continuous. In particular, $\boldsymbol{B}$ and $\boldsymbol{C}$ map $\mathcal{M}_{0}$ to $C\left(\mathbb{R}_{0}^{+}\right)$. Moreover, $x \mapsto A(y-x)=\hat{C}(x-y)-\hat{C}(y-x)$ is continuously differentiable with bounded derivative whenever $x \neq y$. For the case $x=y$, note that $A(z)=\hat{C}(z)\left(\mathrm{e}^{\beta z}-1\right)$, and thus $x \mapsto A(x-y)$ is differentiable also at $x=y$ with derivative $\beta \hat{C}(0)$. Altogether, we get that $\partial_{x} K_{b}(x, y)$ is in $C_{b}\left([0, \delta] \times \mathbb{R}_{0}^{+}\right)$, and Proposition 2.1 implies that $p_{t}$ converges regularly to $p_{\infty}$.

It remains to check assumptions (2.6) and (2.7). Clearly,

$$
\boldsymbol{C}\left[p_{\infty}\right](0)=f(0) \int \hat{C}(y) p_{\infty}(y) \mathrm{d} y>0,
$$

which shows that $\alpha$ is determined by the choice in (3.10). Furthermore,

$$
\lim _{x \rightarrow 0} \frac{x^{\alpha-1}}{q(x)}=\lim _{x \rightarrow 0} \frac{\mathrm{e}^{\beta x}-1}{x f(x)}=\frac{\beta}{f(0)}
$$

which shows that

$$
\gamma=\beta \int_{0}^{\infty} \hat{C}(y) p_{\infty}(y) \mathrm{d} y
$$

By Theorem 2.2, we conclude again that the emerging condensate is described by a Gamma distribution on the relevant scale.

\section{Proof of the theorem}

Let $\left(p_{t}\right)$ be a solution to (2.1). We define, for $x \geqslant 0$,

$$
b_{t}(x):=\boldsymbol{B}\left[p_{t}\right](x),
$$

and, for $x>0$,

$$
c_{t}(x)=\boldsymbol{C}\left[p_{t}\right](x)
$$


Then $p_{t}$ solves the time-inhomogenous linear equation

$$
\partial_{t} p_{t}(x)=b_{t}(x) p_{t}(x)+x^{\alpha} c_{t}(x)
$$

with initial condition $p_{0}$, and thus has the representation

$$
p_{t}(x)=\int_{0}^{t} \mathrm{e}^{\int_{s}^{t} b_{u}(x) \mathrm{d} u} x^{\alpha} c_{s}(x) \mathrm{d} s+\mathrm{e}^{\int_{0}^{t} b_{s}(x) \mathrm{d} s} p_{0}(x) .
$$

We will re-write this representation in a form that will be convenient later on, using the following definitions:

$$
\begin{aligned}
& W_{t}:=\mathrm{e}^{\int_{0}^{t} b_{s}(0) \mathrm{d} s}, \\
& \gamma_{t}(x):=-\frac{1}{x}\left(b_{t}(x)-b_{t}(0)\right) \text { for } x>0 \text {, } \\
& \bar{\gamma}_{s, t}(x):= \begin{cases}\frac{1}{t-s} \int_{s}^{t} \gamma_{r}(x) \mathrm{d} r & \text { if } t>s \\
\gamma_{t}(x) & \text { if } t=s .\end{cases}
\end{aligned}
$$

Then we have

$$
p_{t}(x)=\int_{0}^{t} \frac{W_{t}}{W_{s}} x^{\alpha} c_{s}(x) \mathrm{e}^{-(t-s) x \bar{\gamma}_{s, t}(x)} \mathrm{d} s+W_{t} \mathrm{e}^{-t x \bar{\gamma}_{0, t}(x)} p_{0}(x),
$$

Next, we use our assumptions in order to prove some properties of the quantities $c_{t}$ and $\gamma_{t}$.

Proposition 4.1. Let the assumptions of Theorem 2.2 be fulfilled. Then

(1) $\lim _{t \rightarrow \infty} b_{t}(0)=0$.

(2) For sufficiently large $t, x \mapsto \gamma_{t}(x)$ can be continuously extended to all $x \geqslant 0$. Further, there exists $\delta>0$ and a strictly positive function $\gamma_{\infty}:[0, \delta] \rightarrow \mathbb{R}^{+}$with the property that

$$
\lim _{t \rightarrow \infty} \sup _{x \in[0, \delta]}\left|\gamma_{t}(x)-\gamma_{\infty}(x)\right|=0 .
$$

Moreover, we have that $\gamma_{\infty}(0)=\gamma$, the latter being defined in (2.7).

(3) There exists $\delta>0$ and a continuous function $c_{\infty}:[0, \delta] \rightarrow \mathbb{R}$ such that $c_{\infty}(0)>0$, and

$$
\lim _{t \rightarrow \infty} \sup _{x \in[0, \delta]}\left|c_{t}(x)-c_{\infty}(x)\right|=0 .
$$

Proof. By the comments at the end of Section 2 we have $\boldsymbol{B}\left[p_{\infty}\right](0)=0$ so that by (2.2)

$$
\lim _{t \rightarrow \infty} b_{t}(0)=\lim _{t \rightarrow \infty} \boldsymbol{B}\left[p_{t}\right](0)=\boldsymbol{B}\left[p_{\infty}\right](0)=0,
$$

and (1) is shown. For (2), we define

$$
\gamma_{\infty}(x):=-\frac{1}{x} \boldsymbol{B}\left[p_{\infty}\right](x) \text { for } x>0 .
$$

Since $\boldsymbol{B}\left[p_{\infty}\right](x) q(x)=-x^{\alpha} \boldsymbol{C}\left[p_{\infty}\right](x)$ for all $x>0$, we find that

$$
\lim _{x \rightarrow 0} \gamma_{\infty}(x)=\lim _{x \rightarrow 0} \frac{x^{\alpha}}{x q(x)} \boldsymbol{C}\left[p_{\infty}\right](x)=\gamma
$$

by Assumption (2.7) and the definition of $\gamma$. In particular, $\gamma_{\infty}$ can be continuously extended to the whole half line. We write $b_{\infty}$ for $\boldsymbol{B}\left[p_{\infty}\right]$, and put

$$
s_{t}(x):=b_{\infty}(x)-b_{t}(x) .
$$


Then by (2.2), $x \mapsto s_{t}(x)$ is an element of $C^{1}[0, \delta]$ for some $\delta>0$ and all sufficiently large $t$, and $\lim _{t \rightarrow \infty}\left\|s_{t}\right\|_{C^{1}([0, \delta])}=0$. Furthermore,

$$
\gamma_{t}(x)-\gamma_{\infty}(x)=\frac{1}{x}\left(s_{t}(x)-s_{t}(0)\right)=\int_{0}^{1} s_{t}^{\prime}(r x) \mathrm{d} r
$$

and thus (2) follows. (3) follows directly from Assumption (2.3).

Recall our assumption on the initial condition $p_{0}$ : there exists $\alpha_{0}>0$ and $\eta \in L^{1}$ so that $\eta$ is continuous at $x=0, \eta(0)>0$, and

$$
p_{0}(x)=x^{\alpha_{0}} \eta(x)
$$

for all $x \in[0, \delta]$. Let us also define, for $\beta>0$,

$$
Q_{t}(\beta):=W_{t}^{-1}(t+1)^{1+\beta} .
$$

A direct calculation then gives an expression for $p_{t} / t$ at arguments of the order $1 / t$, namely

$$
\frac{Q_{t}(\beta)}{t} p_{t}\left(\frac{x}{t}\right)=\left(\frac{t+1}{t}\right)^{1+\beta} \mathrm{e}^{-x \gamma_{\infty}(0)}\left(t^{\beta-\alpha} x^{\alpha} J(x, t)+t^{\beta-\alpha_{0}} x^{\alpha_{0}} \mathrm{e}^{x\left(\gamma_{\infty}(0)-\bar{\gamma}_{0, t}(x / t)\right)} \eta\left(\frac{x}{t}\right)\right),
$$

with

$$
J(x, t)=\int_{0}^{t} \frac{Q_{s}(\beta)}{(s+1)^{\beta+1}} c_{s}(x / t) \mathrm{e}^{x\left(\gamma_{\infty}(0)-\frac{t-s}{t} \bar{\gamma}_{s, t}(x / t)\right)} \mathrm{d} s .
$$

Now it is easy to prove the following

Proposition 4.2. Assume that the quantities $\left(b_{t}\right)$ and $\left(c_{t}\right)$ have the properties (1) - (3) from Proposition 4.1, and assume in addition that for $\beta:=\min \left\{\alpha, \alpha_{0}\right\}$, the limit $Q_{\infty}=$ $\lim _{t \rightarrow \infty} Q_{t}(\beta)$ exists in $(0, \infty)$. Then

$$
\lim _{t \rightarrow \infty} \frac{1}{t} p_{t}\left(\frac{x}{t}\right)=\frac{1}{Q_{\infty}} \mathrm{e}^{-x \gamma_{\infty}(0)}\left(1_{\{\beta=\alpha\}} x^{\alpha} \int_{0}^{\infty} \frac{Q_{s}(\beta)}{(s+1)^{\beta}} c_{s}(0) \mathrm{d} s+1_{\left\{\beta=\alpha_{0}\right\}} x^{\alpha_{0}} \eta(0)\right),
$$

and the limit is uniform in $x$ on compact intervals in $(0, \infty)$.

Proof. First we analyse the first summand on the right hand side of (4.5). We show that $J(x, t)$ converges uniformly to

$$
\int_{0}^{\infty} \frac{Q_{s}(\beta)}{(s+1)^{\beta}} c_{s}(0) \mathrm{d} s .
$$

To see this, note that by Proposition $4.1, \bar{\gamma}_{0, t}$ converges uniformly on $[0, \delta]$ to $\gamma_{\infty}$ and by continuity of $\gamma_{\infty}$ in zero we get local uniform convergence of $\bar{\gamma}_{0, t}(x / t)$ to $\gamma_{\infty}(0)$. Hence for each fixed $s$, the quantity

$$
D(s, t):=\sup _{0 \leqslant x \leqslant K}\left|c_{s}(x / t) \mathrm{e}^{x\left(\gamma_{\infty}(0)-\frac{t-s}{t} \bar{\gamma}_{s, t}(x / t)\right)}-c_{\infty}(0)\right|
$$

converges to zero as $t \rightarrow \infty$. The integrand in $J(x, t)$ and $\frac{Q_{s}(\beta)}{(s+1)^{\beta}} c_{s}(0)$ are both bounded by a constant multiple of the integrable function $(s+1)^{-1-\beta}$, uniformly in $x$, on compact intervals. Thus for $M>0$,

$$
\sup _{0 \leqslant x \leqslant K}\left|J(x, t)-\int_{0}^{\infty} \frac{Q_{s}(\beta)}{(s+1)^{\beta}} c_{s}(0) \mathrm{d} s\right| \leqslant \int_{0}^{M} \frac{Q_{s}(\beta)}{(s+1)^{\beta}} D(s, t) \mathrm{d} s+C \int_{M}^{\infty}(s+1)^{-1-\beta} \mathrm{d} s .
$$

The first term above converges to zero by dominated convergence, as $t \rightarrow \infty$. By taking $M \rightarrow \infty$ afterwards, the asymptotic of the first term follows. Using that, uniformly on compact intervals, $\mathrm{e}^{x\left(\gamma_{\infty}(0)-\bar{\gamma}_{0, t}(x / t)\right)} \eta\left(\frac{x}{t}\right) \rightarrow \eta(0)$ we get convergence of the second term. 
We have just proved Theorem 2.2 under the additional assumption that $\lim _{t \rightarrow \infty} Q_{t}(\beta)$ exists and is strictly positive. Since the definition of $Q_{t}(\beta)$ involves the function $b_{t}(0)=$ $\boldsymbol{B}\left[p_{t}\right](0)$ and thus the solution itself, such a condition is not desirable. The main step of our proof consists in showing that existence of $\lim _{t \rightarrow \infty} Q_{t}(\beta)$ already follows from the presence of a condensate, and that its positivity follows from the finiteness of the condensate mass. Actually, we will show a bit more. Note that weak convergence of $p_{t}$ to $\rho \delta_{0}+q \mathrm{~d} x$ implies that

$$
\lim _{\varepsilon \downarrow 0} \lim _{t \rightarrow \infty} \int_{0}^{\varepsilon} p_{t}(x) \mathrm{d} x=\rho .
$$

\section{Proposition 4.3.}

(i): Assume that (1)-(3) from Proposition 4.1 hold, and that the initial condition $p_{0}$ is given by (4.3). Define $Q_{t}(\beta)$ as in (4.4), with $\beta=\min \left\{\alpha, \alpha_{0}\right\}$. Then the following two statements are equivalent:

(a) $\lim _{t \rightarrow \infty} Q_{t}(\beta)$ exists and is finite.

(b) $\left(p_{t}\right)$ exhibits condensation, i.e. (4.7) holds with $\rho>0$.

(ii): If (a) holds with $\lim _{t \rightarrow \infty} Q_{t}(\beta)=0$, and if there exist $\delta>0$ and $T>0$ so that $p_{t}(x) \geqslant 0$ for all $x<\delta$ and all $t>T$, then we have $\rho=\infty$ in (b).

(iii): If (a) holds with $\lim _{t \rightarrow \infty} Q_{t}(\beta)>0$, then for $0<\varepsilon<\delta$,

$$
\lim _{t \rightarrow \infty} \int_{0}^{\varepsilon} p_{t}(x) \mathrm{d} x=\int_{0}^{\infty} \kappa(x) \mathrm{d} x+\int_{0}^{\varepsilon} \frac{x^{\alpha-1} c_{\infty}(x)}{\gamma_{\infty}(x)} \mathrm{d} x,
$$

where $\kappa(x)$ stands for the right hand side of (4.6). Moreover,

$$
\rho=\lim _{K \rightarrow \infty} \lim _{t \rightarrow \infty} \int_{0}^{K / t} p_{t}(x) \mathrm{d} x=\int_{0}^{\infty} \kappa(x) \mathrm{d} x .
$$

Proof. We start by proving parts (ii) and (iii), which also shows that (a) implies (b) in part (i). For part (ii), note that the proof of Proposition 4.2 actually shows that

$$
\lim _{t \rightarrow \infty} \frac{Q_{t}(\beta)}{t} p_{t}\left(\frac{x}{t}\right)=\mathrm{e}^{-\gamma_{\infty}(0) x}\left(1_{\{\beta=\alpha\}} x^{\alpha} \int_{0}^{\infty} \frac{Q_{s}(\beta)}{(s+1)^{\beta}} c_{s}(0) \mathrm{d} s+1_{\left\{\beta=\alpha_{0}\right\}} x^{\alpha_{0}} \eta(0)\right)
$$

under the condition that $\left(Q_{s}(\beta)\right)$ is bounded. If $\lim _{t \rightarrow \infty} Q_{t}(\beta)=0$, this implies that $\lim _{t \rightarrow \infty} \frac{1}{t} p_{t}(x / t)=\infty$ for all $x>0$, and Fatou's lemma together with our positivity assumption gives

$$
\liminf _{t \rightarrow \infty} \int_{0}^{\delta / t} p_{t}(x) \mathrm{d} x=\liminf _{t \rightarrow \infty} \frac{1}{t} \int_{0}^{\delta} p_{t}(x / t) \mathrm{d} x \geqslant \int_{0}^{\delta} \liminf _{t \rightarrow \infty} \frac{1}{t} p_{t}(x / t) \mathrm{d} x=\infty .
$$

This shows (ii). Now assume that $Q_{\infty}>0$.

Then the rightmost equality in (4.9) is proved by observing that

$$
\int_{0}^{K / t} p_{t}(x) \mathrm{d} x=\frac{1}{t} \int_{0}^{K} p_{t}(x / t) \mathrm{d} x \rightarrow \int_{0}^{K} \kappa(x) \mathrm{d} x
$$

as $t \rightarrow \infty$, by Proposition 4.2, and then taking $K \rightarrow \infty$. Let us now write

$$
\tilde{p}_{t}(x):=W_{t} \mathrm{e}^{-t x \bar{\gamma}_{0, t}(x)} p_{0}(x)
$$


for the second term in (4.2). Then for $t$ large enough, we have that

$$
\int_{K / t}^{\varepsilon} \tilde{p}_{t}(x) \mathrm{d} x=\left(\frac{t+1}{t}\right)^{\beta} t^{\beta-\alpha_{0}} Q_{t}(\beta)^{-1} \int_{K}^{t \varepsilon} \mathrm{e}^{-x \bar{\gamma}_{0, t}(x / t)} x^{\alpha_{0}} \eta(x / t) \mathrm{d} x .
$$

Since $\bar{\gamma}_{0, t}(x / t)$ converges uniformly for $x \in[0, t \varepsilon]$ to $\gamma_{\infty}(x / t)$ (provided $\varepsilon<\delta$ ), and since $\gamma_{\infty}(x)>\gamma_{-}>0$ for $x<\delta$, we find that

$$
\lim _{t \rightarrow \infty} \int_{K}^{t \varepsilon} \mathrm{e}^{-x \bar{\gamma}_{0, t}(x / t)} x^{\alpha_{0}} \eta(x / t) \mathrm{d} x \leqslant Q_{\infty}^{-1} \int_{K}^{\infty} \mathrm{e}^{-x \gamma_{-} / 2} x^{\alpha_{0}} \sup _{z \leqslant \varepsilon}|\eta(z)| \mathrm{d} x \rightarrow 0
$$

as $K \rightarrow \infty$. We have shown

$$
\lim _{t \rightarrow \infty} \int_{0}^{\varepsilon} \tilde{p}_{t}(x) \mathrm{d} x=\lim _{K \rightarrow \infty} \lim _{t \rightarrow \infty} \int_{0}^{K / t} \tilde{p}_{t}(x) \mathrm{d} x .
$$

Now we turn to the first term of (4.2). We have

$$
\begin{aligned}
& \int_{0}^{t} \int_{0}^{\varepsilon} \frac{W_{t}}{W_{s}} x^{\alpha} c_{s}(x) \mathrm{e}^{-(t-s) x \bar{\gamma}_{s, t}(x)} \mathrm{d} x \mathrm{~d} s \\
& =\frac{1}{Q_{t}(\beta)} \int_{0}^{t / 2} \int_{0}^{(t-s) \varepsilon} \frac{(t+1)^{1+\beta}}{(t-s)^{1+\alpha}} \frac{Q_{s}(\beta)}{(s+1)^{1+\beta}} z^{\alpha} c_{s}\left(\frac{z}{t-s}\right) \mathrm{e}^{-z \bar{\gamma}_{s, t}\left(\frac{z}{t-s}\right)} \mathrm{d} z \mathrm{~d} s \\
& \quad+\int_{0}^{t / 2} \int_{0}^{\varepsilon} \frac{Q_{t-s}(\beta)}{Q_{t}(\beta)} \frac{(t+1)^{1+\alpha}}{(t-s+1)^{1+\alpha}} x^{\alpha} c_{t-s}(x) \mathrm{e}^{-s x \bar{\gamma}_{t-s, t}(x)} \mathrm{d} x \mathrm{~d} s .
\end{aligned}
$$

For the first integral in (4.11), we dominate the integrand uniformly by a constant multiple of the integrable function

$$
\frac{1}{(s+1)^{1+\beta}} z^{\alpha} e^{-\gamma_{-} z}
$$

(Note that $\beta \leqslant \alpha$ ). If $\beta<\alpha$, then this term converges to zero. In the other case, we apply dominated convergence. In total, we obtain that the first term of (4.11) converges to

$$
1_{\{\beta=\alpha\}} \frac{1}{Q_{\infty}(\beta)} \int_{0}^{\infty} \int_{0}^{\infty} \frac{Q_{s}(\beta)}{(s+1)^{1+\alpha}} z^{\alpha} c_{s}(0) \mathrm{e}^{-z \gamma_{\infty}(0)} \mathrm{d} z \mathrm{~d} s
$$

Since we assumed $Q_{t}(\beta)$ to converge to a strictly positive limit, the integrand of the second integral in (4.11) is bounded by a constant multiple of the integrable function

$$
1_{\{x \leqslant \varepsilon\}} x^{\alpha} e^{-\gamma-s x} .
$$

It therefore converges, by dominated convergence, to

$$
\int_{0}^{\infty} \int_{0}^{\varepsilon} x^{\alpha} c_{\infty}(x) \mathrm{e}^{-s x \gamma_{\infty}(x)} \mathrm{d} x \mathrm{~d} s=\int_{0}^{\varepsilon} \frac{x^{\alpha-1} c_{\infty}(x)}{\gamma_{\infty}(x)} \mathrm{d} x
$$

If we put this together with (4.10), we have completed the proof of (4.8). The first claimed equality of (4.9) now follows by letting $\varepsilon \downarrow 0$ in (4.11). 
Now we prove that $(b)$ implies $(a)$ in (i). Let $\mu_{t}(\varepsilon):=\int_{0}^{\varepsilon} p_{t}(x) \mathrm{d} x$. Then, (4.2) and the definition of $Q_{t}(\beta)$ give

$$
\begin{aligned}
Q_{t}(\beta) \mu_{t}(\varepsilon)= & \int_{0}^{t} \mathrm{~d} s \int_{0}^{\varepsilon} \mathrm{d} x(t+1)^{1+\beta} x^{\alpha} c_{s}(x) \frac{Q_{s}(\beta)}{(s+1)^{1+\beta}} \mathrm{e}^{-(t-s) x \bar{\gamma}_{s, t}(x)} \\
& +\int_{0}^{\varepsilon} \mathrm{d} x(t+1)^{1+\beta} \mathrm{e}^{-t x \bar{\gamma}_{0, t}(x)} x^{\alpha_{0}} \eta(x) \\
= & \int_{0}^{t} \mathrm{~d} s Q_{s} \frac{(t+1)^{1+\beta}}{(s+1)^{1+\beta}(t-s)^{1+\alpha}} \int_{0}^{\varepsilon(t-s)} \mathrm{d} z z^{\alpha} c_{s}\left(\frac{z}{t-s}\right) \mathrm{e}^{-z \bar{\gamma}_{s, t}(z /(t-s))}+ \\
& +\int_{0}^{t \varepsilon} \mathrm{d} z \frac{(t+1)^{1+\beta}}{t^{1+\alpha}} z^{\alpha_{0}} \mathrm{e}^{-z \bar{\gamma}_{0, t}(z / t)} \eta(z / t) .
\end{aligned}
$$

Let now $\varepsilon<\delta$, where $\delta$ is as in Proposition 4.1. Let us also assume that $t^{\prime}$ is sufficiently large so that $\bar{\gamma}_{0, t^{\prime}}(x) \geqslant \gamma_{-} / 2$ for all $0 \leqslant x \leqslant \delta$ and all $t>t^{\prime}$. This is possible since we assumed that $\bar{\gamma}_{0, t}$ converges uniformly to $\gamma_{\infty}>\gamma_{-}$on $[0, \delta]$. Let us furthermore write $\bar{\eta}:=\sup _{0} \leqslant x \leqslant \delta \eta(x)$. Since $\beta \leqslant \alpha_{0}$, the second line of (4.12) is then bounded by

$$
2 \bar{\eta} \int_{0}^{\infty} \mathrm{d} z z^{\alpha_{0}} \mathrm{e}^{-z \gamma_{-} / 2}=: D_{1}<\infty
$$

for all $t$ sufficiently large.

For the first line of (4.12), we fix $q>1$, and for $t>2 q$ we divide the integration range $[0, t]$ of the first integral into $[0, q] \cup[q, t-q] \cup[t-q, t]$. We write

$$
M_{t}:=\sup _{s \leqslant t} Q_{t}(\beta), \quad \bar{c}:=\sup \left\{c_{s}(x): 0 \leqslant x \leqslant \delta, 0 \leqslant s<\infty\right\} .
$$

Note that $\beta \leqslant \alpha$ and thus $(t-s)^{-1-\alpha} \leqslant(t-s)^{-1-\beta}$ when $s \leqslant t-q, q>1$ and $t>2 q$. Thus the integral over $[0, q]$ is bounded by

$$
M_{q} \bar{c} \int_{0}^{q} \mathrm{~d} s \frac{(t+1)^{1+\beta}}{(q+1)^{1+\beta}(t-q)^{1+\beta}} \int_{0}^{\infty} \mathrm{d} z z^{\alpha} \mathrm{e}^{-z \gamma_{-} / 2}=: M_{q} C_{1}(q),
$$

for all $t$ sufficiently large. An elementary estimate shows that

$$
\int_{q}^{t-q} \frac{(t+1)^{1+\beta}}{(s+1)^{1+\beta}(t-s)^{1+\beta}} \mathrm{d} s \leqslant 2 \frac{(t+1)^{1+\beta}}{(t / 2)^{1+\beta}} \int_{q}^{t / 2} s^{-(1+\beta)} \mathrm{d} s \leqslant 2^{5+2 \beta} \beta^{-1} q^{-\beta},
$$

and thus the integral from $q$ to $t-q$ is bounded by

$$
M_{t} \bar{c} \int_{0}^{\infty} \mathrm{d} z z^{\alpha} \mathrm{e}^{-z \gamma_{-} / 2} \int_{q}^{t-q} \mathrm{~d} s \frac{(t+1)^{1+\beta}}{(s+1)^{1+\beta}(t-s)^{1+\beta}}=: M_{t} C_{2} q^{-\beta}
$$

for a suitable constant $C_{2}$ that is independent of $t$ and $q$. Finally, for the integral from $q-t$ to $t$, we undo the change of variable that transformed $x$ into $z=x /(t-s)$. Then this integral is bounded by

$$
M_{t} \int_{t-q}^{t} \mathrm{~d} s \frac{(t+1)^{1+\beta}}{(s+1)^{1+\beta}} \bar{c} \int_{0}^{\varepsilon} x^{\alpha} \mathrm{d} x \leqslant M_{t} q \frac{(t+1)^{1+\beta}}{(t-q+1)^{1+\beta}} \bar{c} \int_{0}^{\varepsilon} x^{\alpha} \mathrm{d} x=: M_{t} C_{3}(q) \varepsilon^{1+\alpha},
$$

where $C_{3}(q)$ can be chosen independently of $t$. We now use that we assumed condensation. Let $\rho>0$ be the mass of the condensate, let $\rho_{1}<\rho$, and let $\varepsilon_{t}$ be the smallest (unique, if $\left.p_{t}>0\right)$ solution of the equation

$$
\int_{0}^{\varepsilon_{t}} p_{t}(x) \mathrm{d} x=\rho_{1}
$$


Then $\mu_{t}\left(\varepsilon_{t}\right)=\rho_{1}$ by definition, and $\lim _{t \rightarrow \infty} \varepsilon_{t}=0$ by condensation. Now we choose $q=\bar{q}$ large enough so that the right hand side of (4.15) is less than $M_{t} \rho_{1} / 3$. Then we choose $\bar{t}$ large enough so that for all $t>\bar{t}$, we have $C_{3}(\bar{q}) \varepsilon_{t}^{1+\alpha} \leqslant \rho_{1} / 3$. Thus for these $t$, the right hand side of (4.16) is less than $M_{t} \rho_{1} / 3$. Writing $D_{2}=M_{\bar{q}} C_{1}(\bar{q})$ for the right hand side of (4.14), an plugging all the parts back into (4.12), we obtain the inequality

$$
Q_{t}(\beta) \rho_{1} \leqslant D_{1}+D_{2}+\frac{2}{3} M_{t} \rho_{1}
$$

valid for all $t>\bar{t}$. Then, for all $t>\bar{t}$, we get

$$
M_{t} \leqslant M_{\bar{t}}+\sup _{\bar{t} \leqslant s \leqslant t} Q_{s}(\beta) \leqslant M_{\bar{t}}+\frac{D_{1}+D_{2}}{\rho_{1}}+\frac{2}{3} \sup _{\bar{t} \leqslant s \leqslant t} M_{s}=M_{\bar{t}}+\frac{D_{1}+D_{2}}{\rho_{1}}+\frac{2}{3} M_{t} .
$$

We conclude that $M_{t} \leqslant 3\left(M_{\bar{t}}+\left(D_{1}+D_{2}\right) / \rho_{1}\right)$ and thus $t \mapsto Q_{t}(\beta)$ is bounded.

Next we show convergence of $\left(Q_{t}(\beta)\right)$. We have

$$
\begin{aligned}
Q_{t}(\beta) \mu_{t}(\varepsilon)= & \int_{0}^{t / 2} \int_{0}^{(t-s) \varepsilon} Q_{s}(\beta)\left(\frac{t+1}{s+1}\right)^{1+\beta} \frac{1}{(t-s)^{1+\alpha}} z^{\alpha} c_{s}\left(\frac{z}{t-s}\right) \mathrm{e}^{-z \bar{\gamma}_{s, t}\left(\frac{z}{t-s}\right)} \mathrm{d} z \mathrm{~d} s \\
& +\int_{0}^{t / 2} \int_{0}^{\varepsilon} Q_{t-s}(\beta)\left(\frac{t+1}{t-s+1}\right)^{1+\alpha} x^{\alpha} c_{t-s}(x) \mathrm{e}^{-s x \bar{\gamma}_{t-s, t}(x)} \mathrm{d} x \mathrm{~d} s \\
& +\int_{0}^{t \varepsilon} \mathrm{d} z \frac{(t+1)^{1+\beta}}{t^{1+\alpha_{0}}} z^{\alpha} \mathrm{e}^{-z \bar{\gamma}_{0, t}(z / t)} \eta(z / t) .
\end{aligned}
$$

For the first integral on the right hand side above, we dominate the integrand uniformly by a constant multiple of

$$
\frac{1}{(s+1)^{1+\alpha}} z^{\alpha} e^{-\gamma_{-} z}
$$

using that $\bar{\gamma}_{s, t}(x)>\gamma_{-}$for all $x \in[0, \varepsilon]$ as long as $t$ is sufficiently large. Hence this integral converges to

$$
\int_{0}^{\infty} \int_{0}^{\infty} \frac{Q_{s}(\beta)}{(s+1)^{1+\alpha}} z^{\alpha} c_{s}(0) \mathrm{e}^{-z \gamma_{\infty}(0)} \mathrm{d} z \mathrm{~d} s=\int_{0}^{\infty} \int_{0}^{\infty} W_{s}^{-1} z^{\alpha} c_{s}(0) \mathrm{e}^{-z \gamma_{\infty}(0)} \mathrm{d} z \mathrm{~d} s
$$

if $\alpha=\beta$, and to zero if $\alpha>\beta$. Further the second term in (4.17) is for $t \geqslant t_{0}$ bounded by

$$
U(\varepsilon)=C_{1} C_{4} 2^{1+\alpha} \int_{0}^{\infty} \int_{0}^{\varepsilon} x^{\alpha} \mathrm{e}^{-s x \bar{\gamma}-(x)} \mathrm{d} x \mathrm{~d} s
$$

which converges to zero as $\varepsilon \downarrow 0$. By similar arguments, the third term converges to

$$
\int_{0}^{\infty} z^{\alpha} \mathrm{e}^{-z \gamma_{\infty}(0)} \eta(0) \mathrm{d} z
$$

if $\beta=\alpha_{0}$, and to zero if $\beta<\alpha_{0}$. Defining

$$
R:=1_{\{\beta=\alpha\}} \int_{0}^{\infty} \int_{0}^{\infty} W_{s}^{-1} z^{\alpha} c_{s}(0) \mathrm{e}^{-z \gamma_{\infty}(0)} \mathrm{d} z \mathrm{~d} s+1_{\left\{\beta=\alpha_{0}\right\}} \int_{0}^{\infty} z^{\alpha} \mathrm{e}^{-z \gamma_{\infty}(0)} \eta(0) \mathrm{d} z,
$$

we see from (4.17) and the assumption that $\lim _{\varepsilon \downarrow 0} \liminf _{t \rightarrow \infty} \mu_{t}(\varepsilon)=\rho>0$ that

$$
\limsup _{t \rightarrow \infty} Q_{t}(\beta) \leqslant \lim _{\varepsilon \downarrow 0} \frac{1}{\liminf _{t \rightarrow \infty} \mu_{t}(\varepsilon)}(R+U(\varepsilon))=\frac{1}{\rho_{0}} R .
$$

Analogously, using that $\lim _{\varepsilon \downarrow 0} \lim \sup _{t \rightarrow \infty} \mu_{t}(\varepsilon)=\rho>0$ we get that

$$
\liminf _{t \rightarrow \infty} Q_{t}(\beta) \geqslant \frac{1}{\rho_{0}} R
$$

Hence $\left(Q_{t}(\beta)\right)$ converges to $R / \rho$. 
The proof of Theorem 2.2 is thus finished. Under its assumptions, Proposition 4.1 guarantees that the solution to (2.1) fulfills equation (4.1) with suitable properties of $b$, $c$ and $\gamma$. Since a finite, nonnegative condensation at $x=0$ is assumed, Proposition 4.3 guarantees that $Q_{\infty}$ exists, is finite and strictly positive. Then, Proposition 4.2 shows the claim of the proof. Note that equation (4.8) confirms that the shape of the bulk density needs to be

$$
q(x)=\frac{x^{\alpha-1} c_{\infty}(x)}{\gamma_{\infty}(x)}=\frac{x^{\alpha} \boldsymbol{C}\left[p_{\infty}\right](x)}{\boldsymbol{B}\left[p_{\infty}\right](x)}
$$

for $x>0$, a fact that already follows from the stationarity of $p_{\infty}$ for equation (2.1). Also, equation (4.9) shows that all of the condensate forms on a scale of $1 / t$ as $t \rightarrow \infty$.

\section{Appendix: proof of proposition 2.1}

Fix $t>0$. Then by our assumptions on $K_{b}$,

$$
\int_{0}^{\infty} \sup _{x \in[0, \delta]}\left|\partial_{x} K_{b}(x, y)\right|\left|p_{t}(y)-q(y)\right| \mathrm{d} y<\infty .
$$

Thus Lebesgue's theorem allows us to differentiate under the integral sign and obtain

$$
\begin{aligned}
\partial_{x}\left(\boldsymbol{B}\left[p_{t}\right](x)-\boldsymbol{B}\left[p_{\infty}\right](x)\right) & =\int_{0}^{\infty} \partial_{x} K_{b}(x, y)\left(p_{t}(y)-q(y)\right) \mathrm{d} y-\rho \partial_{x} K_{b}(x, 0) \\
& =\int_{0}^{\infty} \partial_{x} K_{b}(x, y)\left(p_{t}(\mathrm{~d} x)-p_{\infty}(\mathrm{d} x)\right) .
\end{aligned}
$$

Furthermore,

$$
\boldsymbol{C}\left[p_{t}\right](x)-\boldsymbol{C}\left[p_{\infty}\right](x)=\int_{0}^{\infty} K_{c}(x, y)\left(p_{t}(\mathrm{~d} x)-p_{\infty}(\mathrm{d} x),\right.
$$

with a similar equation for $\boldsymbol{B}\left[p_{t}\right](x)-\boldsymbol{B}\left[p_{\infty}\right](x)$. Thus the claim will be shown once we prove the following lemma:

Lemma 5.1. Let $\left(p_{t}\right)$ and $p_{\infty}=\rho \delta_{0}+q(x) \mathrm{d} x$ fulfill the assumptions of Proposition 2.1. Then we have

$$
\lim _{t \rightarrow \infty} \sup _{0 \leqslant x \leqslant \delta}\left|\int_{0}^{\infty} h(x, y)\left(p_{t}(\mathrm{~d} y)-p_{\infty}(\mathrm{d} y)\right)\right|=0
$$

for all $\delta>0$ and all $h \in C_{b}\left([0, \delta] \times \mathbb{R}_{0}^{+}\right)$.

Proof. First we rewrite

$$
\begin{aligned}
\int_{0}^{\infty} h(x, y)\left(p_{t}(y)-q(y)\right) \mathrm{d} y-\rho h(x, 0)= & \int_{0}^{\infty}(h(x, y)-h(x, 0))\left(p_{t}(y)-q(y)\right) \mathrm{d} y \\
& +h(x, 0)\left(\int_{0}^{\infty}\left(p_{t}(y)-q(y)\right) \mathrm{d} y-\rho\right)
\end{aligned}
$$

By the weak convergence of $p_{t} \mathrm{~d} x$ to $q \mathrm{~d} x+\rho \delta_{0}$, the second line of (5.1) converges to zero uniformly in $x \in[0, \delta]$. For the first line, we fix $\varepsilon>0$. Since $h$ is continuous, it is uniformly continuous on compact intervals. Therefore there exists $\tilde{\delta}>0$ so that for all $x \in[0, \delta]$ and all $y \in[0, \tilde{\delta}]$, we have $|h(x, y)-h(x, 0)|<\varepsilon$. Thus

$$
\left|\int_{0}^{\tilde{\delta}}(h(x, y)-h(x, 0))\left(p_{t}(y)-q(y)\right) \mathrm{d} y\right| \leqslant \varepsilon \int_{0}^{\tilde{\delta}}\left|p_{t}(y)\right|+|q(y)| \mathrm{d} y .
$$


By decreasing $\tilde{\delta}$ if necessary and using condition (2.5), we find that the latter integral is bounded by a constant $C$, and hence (5.2) is bounded by $C \varepsilon$ uniformly in $x \in[0, \delta]$. Note that for sufficiently large $t$ this would follow already from weak convergence of $p_{t}$ if we had assumed that $p_{t}$ is nonnegative. For the remaining part of the first line of (5.1), we estimate

$$
\int_{\tilde{\delta}}^{\infty}(h(x, y)-h(x, 0))\left(p_{t}(y)-q(y)\right) \mathrm{d} y \leqslant 2\|h\|_{\infty} \int_{\tilde{\delta}}^{\infty}\left|p_{t}(y)-q(y)\right| \mathrm{d} y .
$$

By assumption (2.4), this integral tends to zero uniformly in $x$. Putting (5.2) and (5.3) together, we finally get that

$$
\limsup _{t \rightarrow \infty} \sup _{0 \leqslant x \leqslant \delta}\left|\int_{0}^{\infty}(h(x, y)-h(x, 0))\left(p_{t}(y)-q(y)\right) \mathrm{d} y\right| \leqslant C \varepsilon
$$

and the claim follows since $\varepsilon>0$ was arbitrary.

\section{REFERENCES}

[1] Buffet, E.; P. de Smedt, and J.V. Pulé (1984) On the dynamics of Bose-Einstein condensation. Annales de l'Institut Henri Poincaré (C) Analyse non linéaire. 1 413-451.

[2] Buffet, E.; P. de Smedt, P. and J.V. Pulé (1984) The dynamics of the open Bose gas. Ann. Physics. 155 269-304.

[3] Chatterjee, S. and P. Diaconis (2014) Fluctuations of the Bose-Einstein condensate. Jour. of Physics A: Mathematical and Theoretical 47 (8) 085201.

[4] Dereich, S. (2016) Preferential attachment with fitness: unfolding the condensate. Electron. J. Probab. 21, No. 3, 1-38.

[5] Dereich, S. and P. Mörters (2013) Emergence of condensation in Kingmans model of selection and mutation. Acta Appl Math 127 17-26.

[6] Dereich, S.; C. Mailler and P. Mörters. (2017) Nonextensive condensation in reinforced branching processes. Ann. Appl. Probab. 27 2539-2568.

[7] Escobedo, M. and S. Mischler. (1999) Equation de Boltzmann quantique homogene: existence et comportement asymptotique. C. R. Acad. Sci. Paris 329 Serie I (1999) 593-598.

[8] Escobedo, M. and S. Mischler (2001) On a quantum Boltzmann equation for a gas of photons. J. Math. Pures Appl. 80 471-515.

[9] Escobedo, M., S. Mischler and J.J.L. Velazquez (2004) Asymptotic description of Dirac mass formation in kinetic equations for quantum particles. Journal of Differential Equations 202 208-230.

[10] Escobedo, M. and J.J.L Velazquez (2015) Finite time blow-up and condensation for the bosonic Nordheim equation. Invent. Math. 200 761-847.

[11] Kingman, J.F.C. (1978) A simple model for the balance between selection and mutation. J. Appl. Prob. 15 1-12.

[12] Lu, X. (2004) On isotropic distributional solutions to the Boltzmann equation for Bose-Einstein particles. J. Stat. Phys. 116 1597-1649.

[13] Lu, X. (2005) The Boltzmann equation for Bose-Einstein particles: Velocity concentration and convergence to equilibrium. J. Stat. Phys. 119 1027-1067.

[14] Park, S.-C. and J. Krug. (2008) Evolution in random fitness landscapes: the infinite sites model. J. Stat. Mech. Theory Exp. no. 4, P04014, 29pp.

[15] Spohn, H. (2010) Kinetics of the Bose-Einstein condensation. Physica D: Nonlinear Phenomena 239 627-634.

[16] Yuan, L. (2017) A generalization of Kingman's model of selection and mutation and the Lenski experiment. Mathematical Biosciences 285 61-67. 